\section{ALSCAL: A nonmetric multidimensional scaling program with several individual-differences options}

\section{FORREST W. YOUNG, YOSHIO TAKANE, and ROSTYSLAW LEWYCKYJ \\ University of North Carolina \\ Chapel Hill, North Carolina 27514}

ALSCAL is a nonmetric (or metric) multidimensional scaling (MDS) program with a number of individualdifferences options unavailable in other nonmetric MDS programs. This is the only program which incorporates individual-differences MDS models (Carroll \& Chang, 1970; McGee, 1968) with multidimensional unfolding (MDU) models (Coombs, 1964; Young, 1972) and nonindividual-differences MDS models (Guttman, 1968; Kruskal, 1964; Shepard, 1962; Torgerson, 1952). ALSCAL consolidates most of the important developments in multidimensional scaling into a single program.

ALSCAL uses the $A$ lternating $L$ east $S$ quares approach to scaling proposed by Takane, Young, and de Leeuw (1977), as improved by Young, Takane, and Lewyckyj (in press). ALSCAL is capable of performing a wide range of analyses, including (but not limited to) analyses equivalent to those performed by SSA-1, SSAR-1, and MINISSA (Lingoes, 1973), MDSCAL (Kruskal, 1964), KYST (Kruskal, Young, \& Seery, Note 1), POLYCON (Young, 1973), INDSCAL (Carroll \& Chang, 1970), TORSCA (Young, 1968), EMD, CEMD, and DEMD (McGee, 1968), and ASYMSCAL (Baker, Young, \& Takane, Note 2; Young, Note 3).

ALSCAL is suitable for any type of two- or threeway data (rectangular or square, symmetric or asymmetric, conditional or unconditional, replicated or unreplicated, with or without missing data) that may be measured at the nominal, ordinal, interval, or ratio level of measurement (or may be binary).

ALSCAL permits the analysis of an unlimited number of points or subjects in as many as six dimensions. This is the fastest general-purpose MDS program currently available, being 2 to 15 times as fast as the programs mentioned above.

Data and Models. ALSCAL accepts data that are either two- or three-way and that concern objects or events from one, two, or three distinct sets. Given below are the specific types of data appropriate to ALSCAL, examples of these data, and the types of analyses that can be performed for these data.

Data concerning objects from one set must represent some relation between pairs of objects in the set. These are termed two-way one-mode data, referring to the fact that a single set of objects is being paired with itself. For this type of data the analysis performed by ALSCAL corresponds to the nonindividual-differences MDS proposals mady by Kruskal (1964), Shepard (1962), and Torgerson (1952). An example of the data appropriate to this type of analysis is judgments of the similarity of various automobiles to each other. These data are contained in a single square matrix, with rows and columns representing the objects and entries representing the degree of relation between the objects. The data may be symmetric or asymmetric and may have missing elements. ALSCAL represents these objects (automobiles) as points in a multidimensional euclidean space.

ALSCAL can analyze two different types of data concerning objects from two sets. One of the types, termed two-way two-mode data, is data that represents the degree of relation between objects from two distinct sets. For this type of data the analysis performed by ALSCAL corresponds to the nonindividual-differences MDU proposal made by Coombs (1964). Examples of data for this type of analysis are judgments of preference for automobiles from several subjects (representing the degree of relation between automobiles and subjects) or ratings on several attribute rating scales of the degree of attribute present in an automobile (representing the degree of relation between the rating scales and the automobiles). Such data are contained in a single rectangular matrix, with rows representing one set of items (say rating scales) and columns representing the other set (automobiles). This matrix may have missing elements. ALSCAL represents both sets of objects (scales and automobiles or subjects and automobiles) as points in a single multidimensional euclidean space.

The second type of two-mode data that ALSCAL can analyze is three-way two-mode data. These are data for which there are several observations of the relation between pairs of objects in one set. Analyses performed by ALSCAL on these data correspond to the individualdifferences MDS proposals advanced by Carroll and Chang (1970) and McGee (1968). An example of appropriate data is judgments of automobile similarity obtained from several subjects; the objects being judged (automobiles) are one of the modes, and the subjects are the other. These data are contained in several square matrices, there being one matrix for each subject, with each matrix having rows and columns for the objects being judged. Each matrix may be symmetric or asymmetric, and there may be any pattern of missing data.

ALSCAL can analyze three-way two-mode data in any of three distinct ways. First, ALSCAL can scale only the objects, in which case the objects are represented as points in a multidimensional euclidean space and the subjects are treated as replications. This analysis parallels McGee's (1968) treatment of individual differences in response style. Second, ALSCAL can scale both the objects and the subjects, in which case the objects are represented as points in a multidimensional euclidean 
space and the subjects are represented as vectors of weights in a second multidimensional space. This analysis corresponds to Carroll and Chang's (1970) treatment of individual differences in perception and cognition. Third, the two types of analyses can be combined so that both types of individual differences are permitted.

The final type of information appropriate to ALSCAL is three-way three-mode data: data for which there are several observations of the relation between pairs of objects where the objects in the pair are from two distinct sets. The analyses performed by ALSCAL on this type of data correspond to individual-differences versions of Coombs' (1964) MDU model, a type of analysis that has not been previously proposed. An example of data for these analyses involves several subjects rating, on several attribute rating scales, the degree of each attribute present in various automobiles. Such data are contained in several rectangular matrices, there being one matrix for each subject, and each matrix having (say) rows for rating scales and columns for automobiles. The matrices may have any pattern of missing data.

ALSCAL can perform three distinct types of analyses of three-way three-mode data. One of the analyses applies McGee's notion of individual differences to the MDU situation. The rating scales and the objects are both represented as points in a single multidimensional euclidean space, and the subjects are simply treated as replications. Thus, this type of analysis permits individual differences in response bias. The second kind of analysis applies Carroll and Chang's notion of individual differences to the MDU model. In this case the rating scales and objects are still represented as points in a single multidimensional euclidean space, but the subjects are represented as vectors in a second multidimensional space. Here we are permitting individual differences in perception and/or cognition. The third type of analysis applies both individual-differences notions to the MDU model, permitting differences in response style as well as in perception and cognition.

In any situation in which asymmetric data are permitted, ALSCAL can apply an asymmetric version of the euclidean model. If the data are two-way, ALSCAL fits the asymmetric model discussed by Baker, Young, and Takane (Note 2) and Young (Note 3). If the data are three-way, ALSCAL can fit either a replicated or an individual-differences version of their model.

For all types of data and for all models, it is not necessary to assume that all elements of the data matrix are comparable, that is, that all are measured on the same scale or by the same measuring device. In some cases it is obvious that some data elements cannot be meaningfully compared to other elements. For example, when several people rank order automobiles according to preference, there is no reason to believe that a rank of 5 from one subject bears any particular relation to another subject's 5 . We should assume that a particular rank is comparable only to the other ranks in its own rank ordering. ALSCAL can partition the data matrix into subsets of elements that are comparable, where the subsets correspond to rows of data or to matrices of data.

For all types of data and for all models, ALSCAL accepts any type of missing data. There is no requirement that the data matrices be complete.

ALSCAL, then, can scale the objects (and optionally the subjects) in spaces defined by the several models just discussed. The scaling is done so that the distances between the objects correspond, in a least squares sense, to a transformation of the data. The data transformation must fulfill two requirements. (1) The measurement characteristics of the data must be strictly maintained, and (2) the transformation must yield values that are a least squares fit to the squared distances. Least squares transformations are provided which permit the data to be measured at the nominal, ordinal, interval, or ratio levels of measurement, or to be binary. The transformations may be monotonic, linear, or polynomial, or they may be simply category preserving, but in all cases the transformations are least squares. Whatever type of transformation is chosen, it is performed only over those elements that are directly comparable. Thus, different transformations are obtained for each subset of comparable observations. The user may specify that all observations are in a single set, that each matrix of observations forms a subset, or that each row of observations forms a subset.

ALSCAL ordinarily computes those scale values for the objects (and subjects) which best reproduce the chosen transformation of the data. The user can specify, however, that some of the scale values be fixed to values read in at the beginning of the analysis. In this case ALSCAL computes the best values for the remaining parameters. This is useful, for example, when one wants to perform an external unfolding (Carroll, 1972) or when one already has scaled the objects and wishes to compute only subject weights.

Input. The job deck consists of control cards, data matrices, and (optionally) initial configuration matrices. Control cards describe the nature of the data, the details of the analysis, and the desired output. The data matrices may be two- or three-way and triangular, square, or rectangular. Input may be from disk or tape. There may be any pattern of missing data.

Output. Standard output includes a listing of the control cards, the derived configuration (stimulus coordinates and, if appropriate, weights), and the STRESS of the solution. Optional output includes plots of the several spaces, plots of the transformations, and plots of the goodness of fit. (Plots are produced on the printer, hence no special plotting equipment is needed.) Optional output also includes listings of the input data, the transformed data, the derived distances, and the history of the iterative process. The derived parameter values may be punched, if desired. 
Limitations. There is no limit on the number of points, the number of subjects, nor the total number of observations, other than that imposed by the total memory available at the user's computer installation. The maximum number of dimensions is limited to six.

Versions. There are three versions of ALSCAL: ALSCAL-D is a dynamic core allocation version written entirely in FORTRAN IV except for the main routine, which is written in the IBM assembler language. This version cannot be run on non-IBM-compatible equipment unless the main routine is rewritten in the appropriate assembler language. ALSCAL-F is a fixed core version written entirely in FORTRAN IV. While this version is easily transportable to non-IBM machines, the fixed core nature of the program places an upper limit on the total number of observations that can be analyzed. The exact value of the limit depends on the specific computer installation. ALSCALS is a SAS (Statistical Analysis System) procedure version written entirely in FORTRAN IV. However, this version is executable only by SAS, thus SAS must already be implemented at the computer installation in order for ALSCAL-S to run. The syntax and file structure of ALSCAL-S is in every way compatible with SAS.

Availability. Documentation and a computer tape containing the source code, test data, and test data output for each version of ALSCAL are available from Forrest W. Young, Psychometric Laboratory, University of North Carolina, Davie Hall 013-A, Chapel Hill, North Carolina 27514.

\section{REFERENCE NOTES}

1. Kruskal, J. B., Young, F. W., \& Seery, J. B. How to use KYST, a very flexible program to do multidimensional scaling and unfolding. Unpublished manuscript, Bell Telephone Laboratories, 1973.

2. Baker, R. F., Young, F. W., \& Takane, Y. An asymmetric Euclidean model An alternating least squares method with optimal scaling features. Manuscript submitted for publication, 1978.

3. Young, F. W. An asymmetric Euclidean model for multi-process asymmetric data. U.S.-Japan Seminar on Multidimensional Scaling, 1975.

\section{REFERENCES}

CARroll, J. D. Individual differences and multidimensional scaling. In R. N. Shepard, A. K. Romney, \& S. Nerlove (Eds.), Multidimensional scaling: Theory and applications in behavioral sciences (Vol. 1). New York: Academic Press, 1972.

Carroll, J. D., \& Chang, J. J. Analysis of individual differences in multidimensional scaling via an $\mathrm{N}$-way generaliza. tion of "Eckart-Young" decomposition. Psychometrika, 1970, 35, 238-319.

Coomss, C. H. A theory of data. New York: Wiley, 1964.

Guttman, L. A general nonmetric technique for finding the smallest coordinate space for a configuration of points. Psychometrika, 1968, 33, 469-506.

KRUSKal, J. B. Nonmetric multidimensional scaling. Psychometrika, 1964, 29, 1-27, 115-129.

Lingoes, J. C. The Guttman-Lingoes nonmetric program series. Ann Arbor, Mich: Mathesis Press, 1973.

MCGEE, V. C. Multidimensional scaling of $n$ sets of similatity measures: A nonmetric individual differences approach. Multivariate Behavioral Research, 1968, 3, 233-248.

ShePARD, R. N. The analysis of proximities:-Multidimensional scaling with an unknown distance function. I and 11. Psychometrika, 1962, 27, 125-140, 219-246.

Takane, Y., Young, F. W., \& de Leeuw, J. Nonmetric individual differences multidimensional scaling: An alternating least squares method with optimal scaling features. Psychometrika, 1977, 42, 7-67.

Torgerson, W. S. Multidimensional scaling: I. Theory and method. Psychometrika, 1952, 17, 401-419.

YounG, F. W. TORSCA-9: A FORTRAN IV program for nonmetric multidimensional scaling. Behavioral Science, $1968,13,343-344$.

Younc, F. W. A model for polynomial conjoint analysis algorithms. In R. N. Shepard, A. K. Romney, \& S. Nerlove (Eds.), Multidimensional scaling: Theory and Applications in the behavioral sciences (Vol. 1). New York: Academic Press, 1972.

Young, F. W. POLYCON: A program for multidimensionally scaling one-, two-, or three-way data in additive, difference, or multiplicative spaces. Behavioral Science, $1973,18,152-155$.

Young, F. W., Takane, Y., \& LEWYCKYJ, R. Three notes on ALSCAL. Psychometrika, in press.

(Accepted for publication March 20, 1978.) 